\title{
Estilos de Paternidad y Conducta Violenta de Jóvenes de la Educación Media
}

\author{
Parenting Styles and Violent Behavior in Secondary Education
}

\author{
Mg. Miriam Silberstein
}

\section{RESUMEN}

El propósito del estudio ha sido determinar si los estilos de paternidad se relacionan con la conducta violenta de jóvenes de la Educación Media, describiendo actitudes violentas de padres y estudiantes, caracterizando los estilos que generan violencia y determinando la percepción que jóvenes y docentes tienen acerca de la violencia familiar, identificando hábitos que incrementan la violencia. Los datos fueron recolectados a través de instrumentos construidos teniendo en cuenta la matriz de operacionalización de las variables del problema. La aplicación a la población de alumnos con muestra representativa intencionalmente seleccionada, se realizó en octubre de 2010 en cuatro colegios de gestión privada de Asunción. Entre los resultados se encontraron coincidencias en los tipos de agresión verbal y física empleados por los jóvenes. Se concluyó que tanto los padres como los hijos utilizan actitudes violentas físicas y verbales; se identificaron los estilos autoritario y permisivo como generadores de conductas violentas; la percepción de los jóvenes y de los docentes es que existe violencia intrafamiliar y los hábitos que favorecen dichas conductas se traducen en frecuentar entornos inadecuados y el consumo de drogas. Resulta propicio el estilo autoritario y el estilo permisivo para fomentar conductas violentas en los jóvenes.

Palabras clave: estilos de paternidad, relación intrafamiliar, conducta violenta, violencia familiar, violencia juvenil. 


\begin{abstract}
The purpose of the study was to determine if parenting styles are related to violent behavior in secondary students by describing violent attitudes of parents and students, characterizing the parenting styles that produce violence, and determining the perception that students and teachers have of domestic violence by identifying the habits that increase it. The research was conducted in October 2010 in four private schools in Asunción. Findings indicated coincidences in the types of verbal and physical aggression used by students. It was concluded that both parents and children use physical and verbal violent attitudes. The authoritarian and permissive styles were identified as generating violent behavior. The perception by students and teachers is that domestic violence exists and the habits that favor such behaviors are related to visiting inappropriate places and drugs use. Both, authoritarian and permissive styles are favorable for creating violent behaviors in students.
\end{abstract}

Keywords: parenting styles, intrafamily relations, violent behavior, domestic violence, youth violence. 


\section{UNIBE

\section{INTRODUCCIÓN}

Son varios los factores que inciden en la conducta violenta de los jóvenes, en este caso el contexto educativo, actos violentos cometidos por los adolescentes hacia otros adolescentes del mismo colegio y de otras instituciones. Tal es el caso del joven del colegio San José de gestión privada de la ciudad de Asunción (Paraguay), que fue agredido brutalmente y que debió ser hospitalizado y operado varias veces del páncreas en el año 2007, así como otros casos menos graves, pero no exentos de violencia.

Estos jóvenes son de un contexto socioeconómico de medio superior a superior, lo que significa que la pobreza no es un factor preponderante en la generación de violencia. El presente trabajo está dedicado a un tema que reviste gran interés para los padres de familia, para los miembros de la familia en general, así como para profesores, educadores y para la sociedad en su conjunto.

Entonces, si se pregunta cuál es el generador de esta violencia, no puede ser dejado fuera de estas consideraciones: el matrimonio y las relaciones intrafamiliares constituyen el fundamento de la sociedad.

En el año 2001, la Asamblea General de las Naciones Unidas (NN.UU.) solicitó su Secretario General. la realización de un estudio en profundidad sobre el tema de la violencia contra los niños, niñas y adolescentes en la región de América Latina, Cuba y República Dominicana con la participación de Paraguay.

El informe analiza la violencia en hogares y familias; en la escuela y otros entornos educativos; en la comunidad y en las calles; en las instituciones y en ámbitos laborales. En el informe se reconoce que la conducta violenta se aprende y la primera oportunidad para aprender a comportarse agresivamente surge en el hogar, observando e imitando la conducta agresiva de los padres, madres así como de otros familiares.

Este tema tan vigente en la actualidad en varios países y de tanta importancia, lleva a reflexionar que en el país no se han encontrado investigaciones que relacionen los estilos de paternidad y la conducta violenta de los jóvenes.

Los términos claves que determinan las variables del estudio se definen como: 
Estilos de paternidad: estilos de paternidad son el conjunto de actitudes, sentimientos y patrones de conducta que los padres asumen frente al niño y que repercuten en el funcionamiento tanto psicológico como social de los hijos (Baumrind, 1971).

Conducta violenta: forma de ejercer el poder mediante el empleo de la fuerza física, psicológica, económica y política (Corsi, 1994).

Los estilos de paternidad y la violencia en jóvenes constituyen un campo de acción del psicopedagogo tanto en la capacitación a docentes, consejería a los padres y en el entrenamiento a los jóvenes de las habilidades necesarias para interaccionar sin conflictos.

Es importante recalcar que este es un tema de trascendental importancia atendiendo a los diversos sucesos que en la sociedad actual vienen aconteciendo con los alumnos de varias instituciones, y con adolescentes en general, que involucran conductas violentas.

Para el desarrollo del estudio, se trazó como objetivo general: determinar los estilos de paternidad y su prevalencia en la conducta violenta de jóvenes de la educación media de colegios privados de la ciudad de Asunción, y como objetivos específicos: describir algunas de las actitudes violentas que manifiestan los jóvenes de la educación media; identificar los estilos de paternidad que con mayor frecuencia generan conductas violentas; explicar la prevalencia de la relación intrafamiliar en la conducta de jóvenes de la educación media; determinar si la violencia en la conducta de los padres favorece la adquisición de conductas violentas en los jóvenes.

A partir de esto, el justificativo del estudio se respalda en la necesidad de determinar los estilos de paternidad y su prevalencia en la conducta violenta de los jóvenes de la educación media a fin de aportar datos que colaboren en la atención educativa pertinente de los docentes, para los progenitores que al carecer de información relevante, no son conscientes de las consecuencias perjudiciales para la cohesión familiar, y del educando para que logren un nivel de compromiso en la adaptación adecuada a nivel social para alcanzar el desarrollo pleno.

\section{MATERIALES Y MÉTODOS}

Para alcanzar los objetivos propuestos, el tipo de estudio utilizado ha sido descriptivo, porque buscó describir un fenómeno de la realidad y no pretendió dar explicaciones de las causas ni generalizar los hallazgos, y de corte transversal, porque se realizó la recolección de los datos en un 
único momento, durante el mes de octubre de 2010. La investigación no pretendió generalizar los resultados, sino obtener riqueza de información que permita entender el fenómeno. Por eso, el tamaño de la muestra no se fijó a priori sino que se han analizado aquellos casos que han llevado a responder las preguntas formuladas en la investigación.

La población conformada por los jóvenes de la Educación Media de los colegios N 1; N 2; N 3 y N 4 de la ciudad de Asunción. Además, los docentes, profesores guías y padres de los alumnos.

Tal como se expresa, la muestra es intencional, dada por todos los alumnos que han presentado situación de conducta violenta que ameritan seguimiento desde los equipos técnicos institucionales y por ello no requiere ni muestra representativa ni el consiguiente cálculo de muestreo (Hernández Sampieri, et al., 1996).

El criterio de selección que se aplicó fue la apertura de las instituciones al pedido y dentro de ellas la disponibilidad libre -no obligatoria- de los alumnos, docentes y padres encuestados. Se realizó el pedido a ocho directores/as y se consiguió la autorización para las cuatro que conforman la población.

El criterio de inclusión para la muestra han sido todos los alumnos del sexo femenino y masculino cursando el primero, segundo y tercer año del Nivel Medio y cuyas edades fluctúan entre los 15 y 18 años y concurren a las instituciones mencionadas y presentan características de conducta violenta y han quedado excluidos de la muestra los alumnos que no respondieron a las características mencionadas y que concurrían a otras instituciones. 


\section{Cuadro $\mathbf{N}^{\circ} 1$ - Matriz de Operativización de Variables \\ Definición operacional de las variables}

\begin{tabular}{|c|c|c|c|}
\hline VARIABLE & CONCEPTUAL & OPERACIONAL & INDICADORES \\
\hline $\begin{array}{l}\text { Estilos de } \\
\text { Paternidad }\end{array}$ & $\begin{array}{l}\text { Conjunto } \\
\text { de actitudes, } \\
\text { sentimientos } \\
\text { y patrones de } \\
\text { conducta que los } \\
\text { padres asumen } \\
\text { frente al niño y } \\
\text { que repercuten en } \\
\text { el funcionamiento } \\
\text { tanto psicológico } \\
\text { como social de } \\
\text { los hijos infantiles } \\
\text { (Baumrind, 1971) }\end{array}$ & $\begin{array}{l}\text { 1. Demócrata } \\
\text { 2. Autoritario } \\
\text { 3. Permisivo }\end{array}$ & $\begin{array}{l}\text { 1.1. Protección } \\
\text { 1.2. Razonamiento } \\
\text { 1.3. Participación } \\
\text { democrática } \\
\text { 1.4. Buen } \\
\text { comportamiento } \\
\text { 2.1. Hostilidad } \\
\text { verbal } \\
\text { 2.2. Castigo } \\
\text { corporal } \\
\text { 2.3. Estrategias } \\
\text { punitivas } \\
\text { 2.4. Poca } \\
\text { directividad } \\
\text { 3.1. Falta de } \\
\text { supervisión } \\
\text { 3.2. Ignorar mal } \\
\text { comportamiento } \\
\text { 3.3. Carencia } \\
\text { de confianza en } \\
\text { práctica }\end{array}$ \\
\hline $\begin{array}{l}\text { Cond u c t a } \\
\text { violenta }\end{array}$ & $\begin{array}{l}\text { Forma de ejercer el } \\
\text { poder mediante el } \\
\text { empleo de la fuerza } \\
\text { física, psicológica, } \\
\text { económica y política } \\
\text { (Corsi, 1994) }\end{array}$ & $\begin{array}{l}\text { 1. Violencia } \\
\text { verbal } \\
\begin{array}{l}\text { 2. Violencia } \\
\text { física }\end{array}\end{array}$ & $\begin{array}{l}\text { 1.1. Rumores } \\
\text { 1.2. Discriminación } \\
\text { 1.3. Groserías } \\
\text { 1.4. Insultos } \\
\text { 2.1. Amenaza con } \\
\text { arma de fuego } \\
\text { 2.2. Amenaza con } \\
\text { arma blanca } \\
\text { 2.3. Golpes } \\
\text { 2.4. Empujones } \\
\text { 2.5. Robos }\end{array}$ \\
\hline
\end{tabular}


Los datos que se obtuvieron a través de cuestionarios y guías de observación fueron manejados con procedimientos estadísticos a través del ordenador y se organizaron en porcentajes. Los datos no cuantificables que arrojó el estudio a través de la guía de observación que no son precodificado fueron tratados por la categoría emergente y se integran en el análisis de los gráficos según corresponda a la variable comunicada. Las entrevistas semiestructuradas fueron sintetizadas y se presentan los aspectos más significativos de los relatos.

\section{RESULTADOS Y DISCUSIÓN}

Del total de alumnos encuestados, el 21,4\% son considerados alumnos violentos por los docentes.

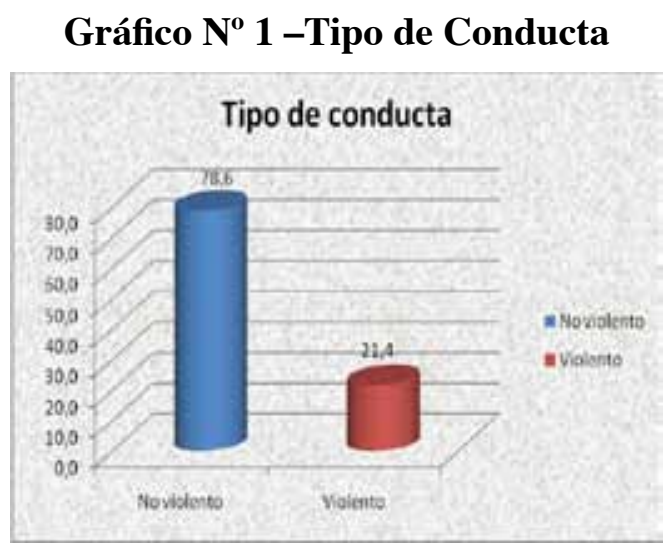

Fuente: cuestionario a los actores de la investigación, 2010. 


\section{ESTILO AUTORITARIO}

\section{Gráfico $\mathbf{N}^{0} 2$ - Hostilidad verbal}

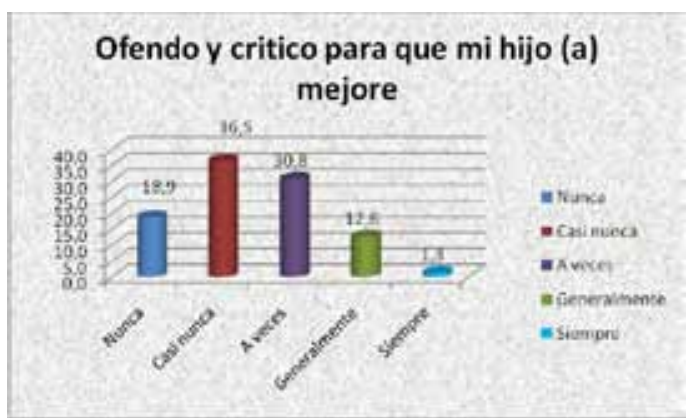

Fuente: cuestionario a los actores de la investigación, 2010.

Con $36,5 \%$, los padres opinaron que "Casi nunca" ofenden y critican para que el hijo mejore. Sin embargo, el 50\% aproximadamente opinó que "A veces" y "Generalmente" lo hacen. Así, sproximadamente el $82 \%$ de los padres han utilizado la ofensa y la crítica para que el hijo mejore.

Gráfico No 3 -Castigo corporal

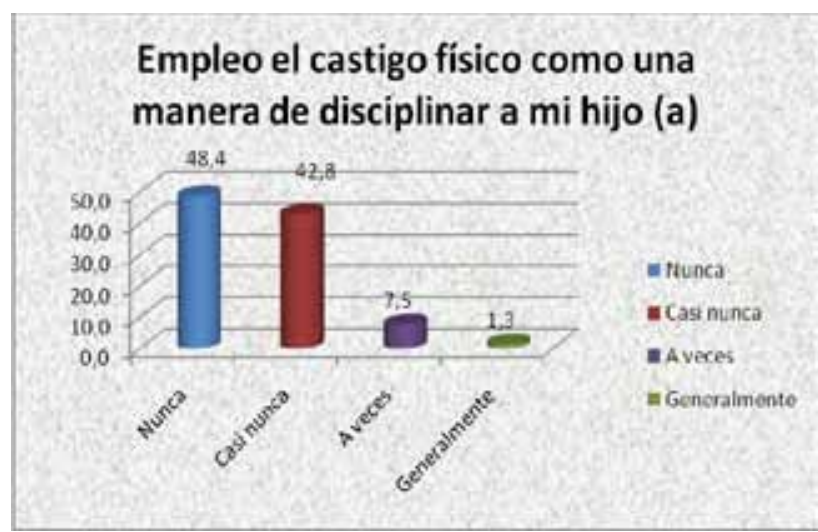

Fuente: cuestionario a los actores de la investigación, 2010.

Con el 48,4\%, los padres opinaron que no emplean el castigo físico con sus hijos y el 42,8\% manifestó que "Casi nunca"; de esto se puede inferir que el castigo físico se utiliza como estrategia disciplinaria en algunos momentos. 


\section{Gráfico No 4 -Poca directividad}

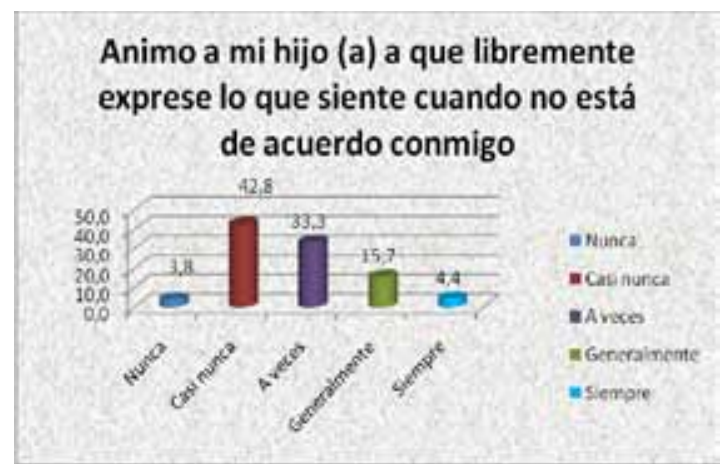

Fuente: cuestionario a los actores de la investigación, 2010.

Aproximadamente el $45 \%$ de los padres opinó que "Nunca" y "Casi nunca" les animan a sus hijos a emitir su desacuerdo con ellos.

ESTILO DEMÓCRATA

\section{Gráfico N 5 -Protección}

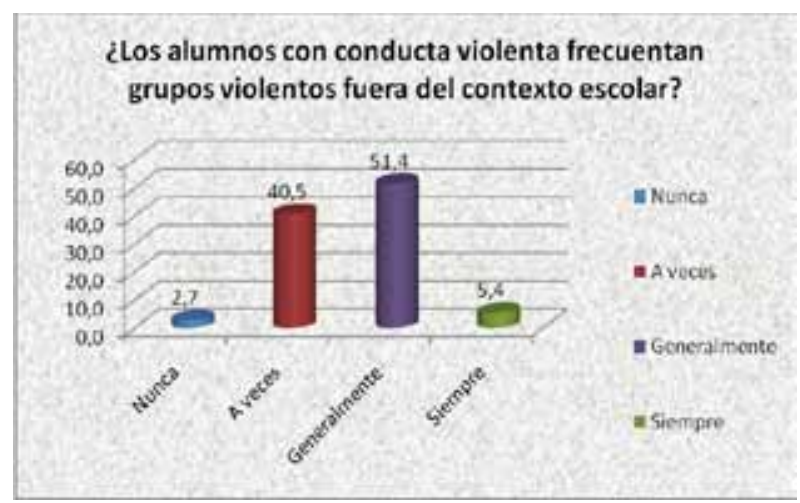

Fuente: cuestionario a los actores de la investigación, 2010.

El 51,4\% de los docentes manifiesta que los alumnos frecuentan grupos violentos fuera del contexto escolar, por lo tanto, no reciben la suficiente protección de sus progenitores, ya que creen que solo el 2,7\% nunca lo hacen. 


\section{Gráfico $\mathbf{N}^{0} 6$-Razonamiento}

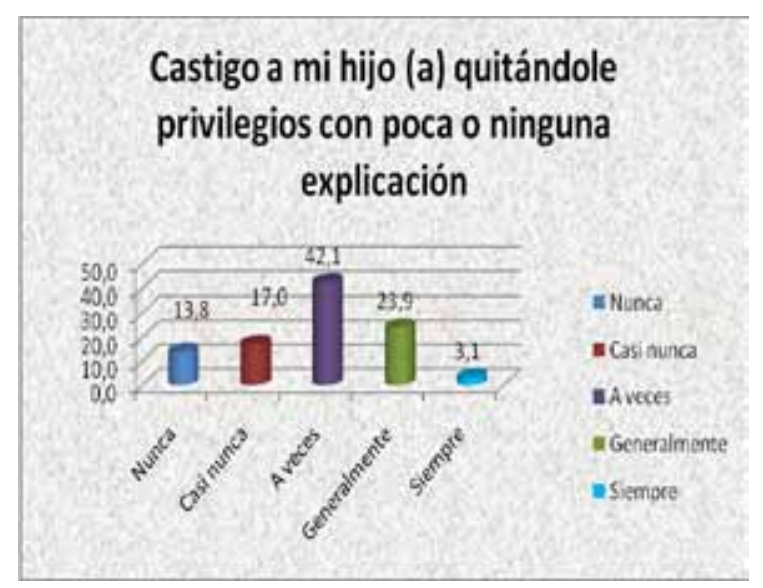

Fuente: cuestionario a los actores de la investigación, 2010.

Se puede inferir que los padres no dan explicaciones de los castigos, ya que los campos "Siempre" y "Generalmente" suman $27 \%$ y el $41,1 \%$, a veces, es decir más del 50\%.

\section{Gráfico $\mathbf{N}^{\circ} 7$-Participación demócrata}

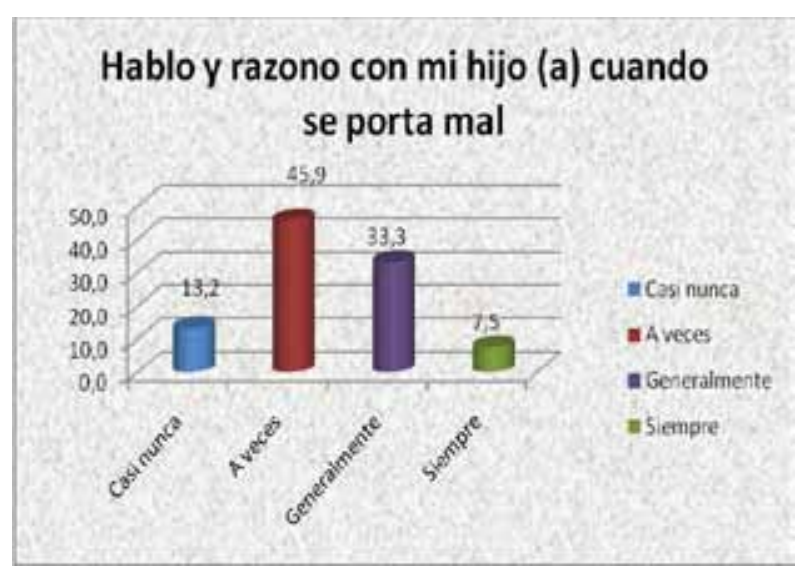

Fuente: cuestionario a los actores de la investigación, 2010.

Aproximadamente el $60 \%$ opinaron que "Casi nunca" y "A veces" hablan y razonan cuando los hijos se portan mal y cerca del $40 \%$ manifestó que 
"Siempre" y "Generalmente" lo hacen. Se infiere que más de la mitad no acostumbran a hablar y razonar con los hijos.

\section{Gráfico No 8 - Buen comportamiento}

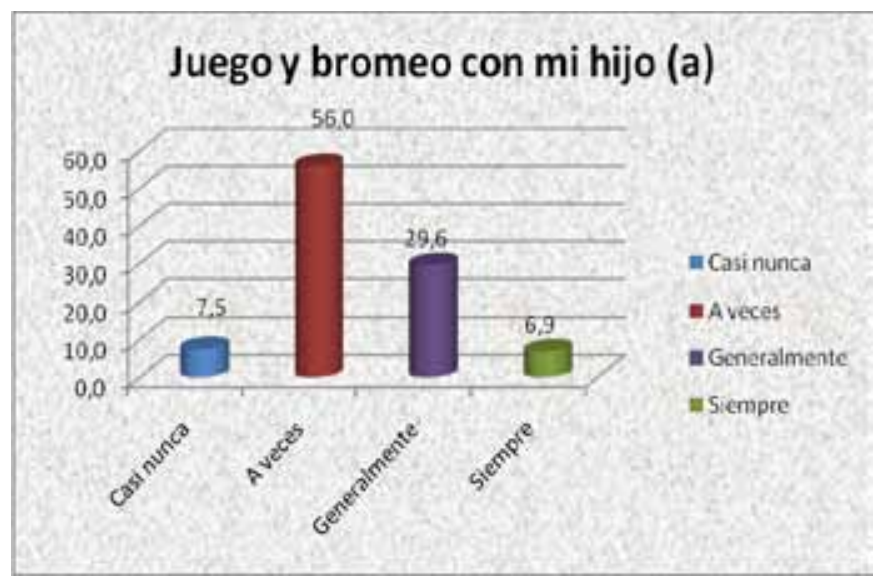

Fuente: cuestionario a los actores de la investigación, 2010.

Más de la mitad de los padres opinó que "A veces" y "Casi nunca" juegan y bromean con los hijos.

EsTILO PERMisIVo

Gráfico N$^{\circ}$ 25- Falta de supervisión

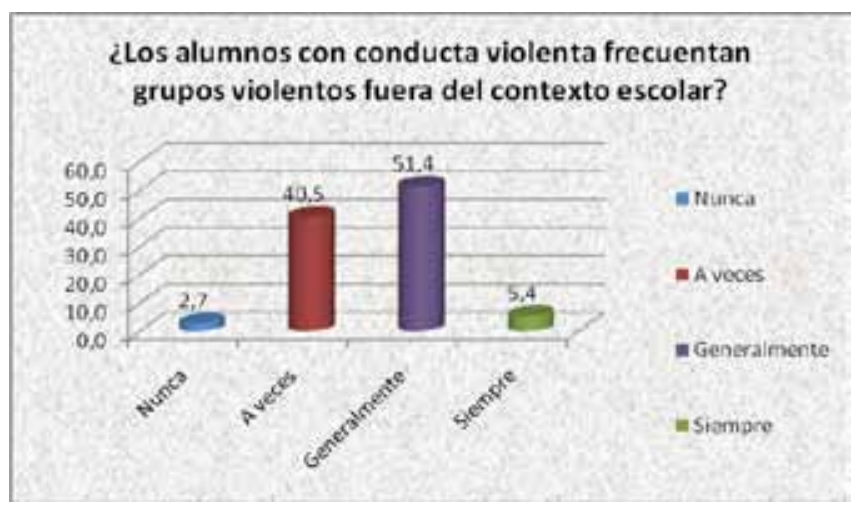

Fuente: cuestionario a los actores de la investigación, 2010. 
Los docentes manifestaron que el 56,8\%, siempre y generalmente los alumnos violentos frecuentan grupos violentos fuera del contexto escolar y el $40,5 \%$ a veces.

\section{Gráfico $\mathbf{N}^{0} 28$-Ignorar mal comportamiento}

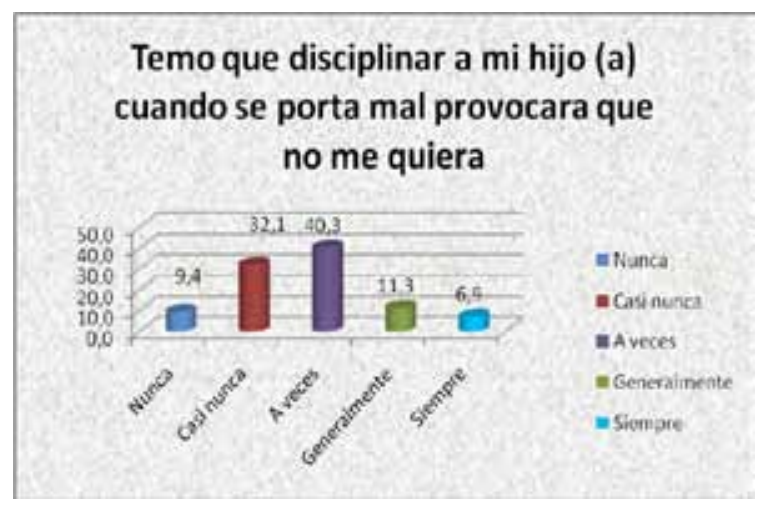

Fuente: cuestionario a los actores de la investigación, 2010.

El 65\% de los padres opinó que a veces, generalmente y siempre tienen temor a disciplinar a sus hijos.

\section{Gráfico No 11 -Falta de límites}

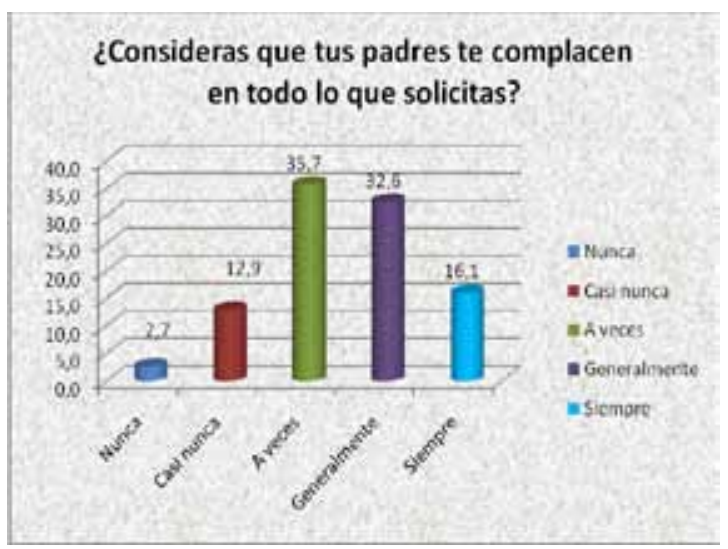

Fuente: cuestionario a los actores de la investigación, 2010.

Con el 48,7\%, los alumnos manifestaron que "Siempre" y "Generalmente" los padres los complacen en todo y con el $35,7 \%$ que a veces lo hacen. 


\section{Gráfico $\mathbf{N}^{0}$ 12: Violencia verbal}

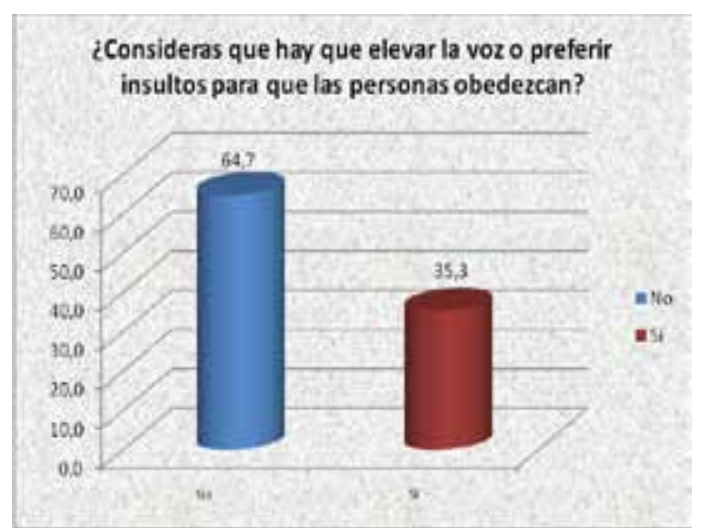

Fuente: cuestionario a los actores de la investigación, 2010.

Los jóvenes opinaron con $64,7 \%$ que no hay que elevar la voz o insultar; mientras el 35,3\% dijo que sí es necesario realizar esa acción para que se obedezca. Se puede inferir que el porcentaje es importante.

\section{Gráfico No 13 - Violencia física}

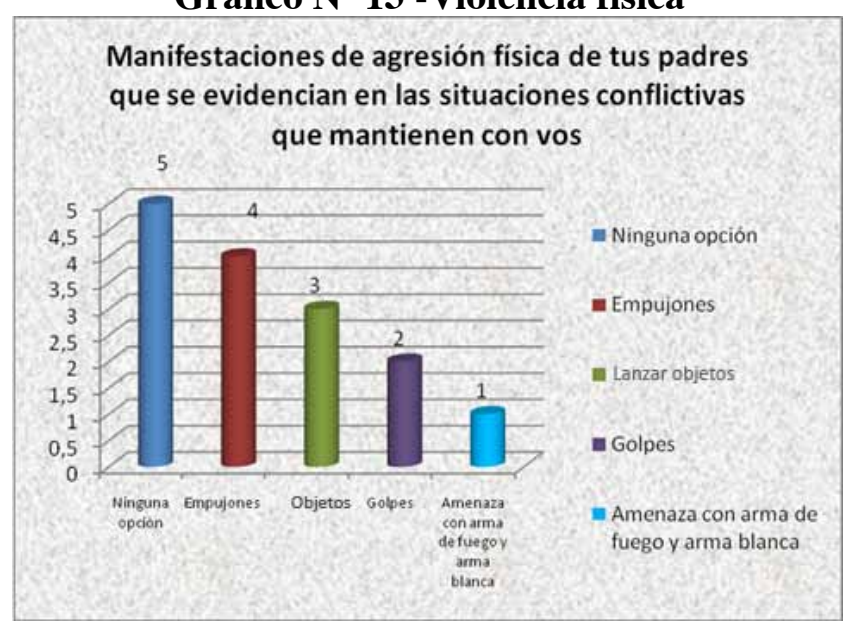

Fuente: cuestionario a los actores de la investigación, 2010. 
Las manifestaciones de agresión física que se evidencian en situaciones conflictivas de padres con hijos son de menor a mayor frecuencia: amenaza con arma de fuego y arma blanca, golpes, lanzar objetos, empujones.

Se puede inferir que los jóvenes reciben con mayor frecuencia, empujones, lanzamiento de objetos, golpes y amenaza con arma de fuego y blanca.

El porcentaje de alumnos violentos alcanzó el 21,4\% a partir de la identificación de los mismos realizadas por los docentes. Con relación al estilo de paternidad demócrata en su indicador de protección los alumnos con conducta violenta que frecuentan grupos violentos fuera del contexto escolar alcanzan el $51,4 \%$, por lo tanto, no reciben la suficiente protección de sus progenitores. Si bien los alumnos manifestaron, con el 41,1\%, que los padres no se desentienden de ellos, el otro $50 \%$ manifestó lo contrario; y con el 64,3\% expresó que siempre se siente amado, el $40 \%$ aproximadamente no dijo siempre. Llamó la atención el alto porcentaje de jóvenes que sienten la falta de amor de sus padres, por lo cual se pudo inferir que habría una falta de protección de los padres hacia sus hijos.

Con relación al estilo demócrata en su indicador razonamiento, se pudo inferir que los padres no dan explicaciones de los castigos, ya que siempre y generalmente suman el $27 \%$ y el $41,1 \%$ a veces, es decir, más del 50\%; aproximadamente el 53\% de los alumnos opinó que los padres toman los errores como forma de aprendizaje, sin embargo, el $45 \%$ aproximadamente manifestó lo contrario.

En el indicador participación demócrata, el 50\% de los padres nunca y casi nunca animan a que sus hijos se expresen en desacuerdo con ellos y el 33,3\% manifestó que a veces; el 69,9\% de los alumnos opinaron que se comunican sus padres adecuadamente con ellos, lo que significa incoherencia con los datos anteriores; y aproximadamente el 60\%, casi nunca y a veces hablan y razonan cuando los hijos se portan mal y el $40 \%$ manifestó que siempre y generalmente lo hacen; se infiere que más de la mitad no acostumbran a hablar y razonar con los hijos.

El indicador buen comportamiento, más de la mitad de los padres opinó que a veces y casi nunca juegan y bromean con los hijos, mientras que el $62,9 \%$ manifestó que a veces son pacientes con los hijos.

Con relación al estilo de paternidad autoritario en su indicador de hostilidad verbal, con el $36,5 \%$ los padres opinaron que casi nunca ofenden y critican para que el hijo mejore, sin embargo, el 50\% aproximadamente opinó que a veces y generalmente lo hacen; las agresiones verbales de 
mayor a menor frecuencia corresponde a insulta, ordena, grita y otros; los padres manifestaron, con un $69,5 \%$, entre generalmente y siempre que gritan y rezongan cuando el hijo se porta mal y el 59,8\%, que nunca y casi nunca emplean violencia verbal para que los hijos obedezcan y el $50 \%$ que lo hace a veces, generalmente y siempre.

En el indicador castigo corporal, si bien el 61,6\% de los alumnos consideraron que no se debe golpear a otros, el 38,4\% opinó que sí, y este es un alto porcentaje; con el 48,4\% los padres opinaron que no emplean el castigo físico con sus hijos y el 42,8\% manifestó que casi nunca, por lo que se infiere que el castigo físico se utiliza como estrategia disciplinaria en algunos momentos. La percepción de los docentes, con el 62,2\% es que los alumnos violentos son sometidos a castigos físicos por los padres. Con el 86,5\%, los docentes manifestaron que nunca presenciaron golpes de los padres a los hijos, y el 13\% opinó que casi nunca y a veces. Se puede inferir que algunas agresiones físicas se han manifestado. Se puede concluir que la percepción de los docentes se puede deber a que si bien el porcentaje ha sido bajo, $13 \%$, haber presenciado manifestaciones violentas de padres a hijos generalizan lo observado.

En el indicador estrategias punitivas, si bien el 32,6\% de los alumnos opinó que nunca son castigados por no seguir la regla del hogar, el 70\% aproximadamente restante se encuentra distribuido en las otras opciones. Se puede inferir que son castigados en demasía con un alto porcentaje. Aproximadamente el $82 \%$ ha utilizado la ofensa y la crítica para que el hijo mejore y el $66 \%$ de los padres opinó que castiga al hijo quitándoles privilegios sin dar explicaciones, lo que podría indicar conductas autoritarias de los padres.

En el indicador poca directividad, aproximadamente el $45 \%$ de los padres opinó que nunca y casi nunca les animan a sus hijos a emitir su desacuerdo con ellos. Aproximadamente el 66\% de los alumnos manifestaron que los padres les responden con atención e interés. Si bien los padres escuchan a sus hijos con interés, no permiten espacios para el disenso.

Con relación al estilo de paternidad permisivo en su indicador falta de supervisión, los docentes manifestaron que el 56,8\%, siempre y generalmente los alumnos violentos frecuentan grupos violentos fuera del contexto escolar y el $40,5 \%$, a veces. Los alumnos opinaron, con el $38,3 \%$, que siempre y generalmente, y con el $33 \%$, a veces que los padres los motivan con pocas presiones. Casi el $50 \%$ de los padres ignora la 
mala conducta de sus hijos. Si bien los padres motivan adecuadamente a sus hijos, se encuentran altos porcentajes de falta de supervisión que coincide con la percepción de los docentes, que manifiestan que los jóvenes violentos frecuentan grupos violentos.

En el indicador ignorar el mal comportamiento, el 65\% de los padres opinaron que a veces, generalmente y siempre tienen temor a disciplinar a sus hijos. Según la percepción de los docentes, los alumnos violentos consumen drogas, con el 78,4\% siempre y generalmente. De todo lo dicho se puede inferir que la falta de disciplina de padres permisivos puede conducir a conductas inadecuadas de parte de los hijos.

En el indicador falta de límites, con el 48,7\% los alumnos manifestaron que siempre y generalmente los padres los complacen en todo, y con el $35,7 \%$, a veces. El 56,6\% de los padres manifestaron que siempre y generalmente consienten a sus hijos, mientras que el 39\%, a veces. Se infiere que los porcentajes de complacencia son muy altos.

Con relación a la conducta violenta en su indicador violencia verbal, las manifestaciones de agresión verbal que se evidencian en situaciones conflictivas de padres con hijos son de menor a mayor frecuencia: otros, grita, ordena, insulta y no respondieron con la mayor frecuencia. Se puede inferir que los jóvenes reciben con mayor frecuencia insultos, órdenes, gritos y otros. Los jóvenes opinaron, con $64,7 \%$, que no hay que elevar la voz o insultar, y el 35,3\% dijo que sí es necesario realizar esa acción para que se obedezca. Se puede inferir que el porcentaje es importante. Los docentes perciben, con un $83,7 \%$, que generalmente y siempre los jóvenes provienen de relaciones intrafamiliares conflictivas. Las actitudes violentas en forma verbal que los jóvenes manifestaron de mayor a menor frecuencia son groserías, insultos, rumores, entredichos y desprecios.

La conducta violenta en su indicador violencia física las manifestaciones de agresión física que se evidencian en situaciones conflictivas de padres con hijos son de menor a mayor frecuencia: amenaza con arma de fuego y arma blanca, golpes, lanzar objetos, empujones. Se puede inferir que los jóvenes reciben con mayor frecuencia empujones, lanzamiento de objetos, golpes y amenaza con arma de fuego y blanca. Los jóvenes opinaron, con $61,6 \%$, que no deben ser golpeadas las personas, y el $38,4 \%$ dijo que sí es necesario realizar esa acción para que se obedezca. Se puede inferir que el porcentaje es importante. Las actitudes violentas en forma física son de mayor a menor: empujones, golpes, robos, 
amenaza con arma blanca y de fuego. La percepción de los docentes, con el $62,2 \%$, es que los alumnos violentos son sometidos a castigos físicos por los padres.

Cuadro $\mathbf{N}^{0} 2$ - Guía elaborada para comparar los resultados obtenidos a partir de las respuestas de los entrevistados.

\begin{tabular}{|c|c|c|l|r|c|}
\hline \multicolumn{7}{|c|}{ GUÍA DE OBSERVACIÓN } \\
\hline \multirow{2}{*}{ DURANTE TRES (3) DÍAS } \\
\hline \multirow{2}{*}{$\mathbf{N} 2$} & Violencia verbal & \multicolumn{1}{c|}{ Violencia física } \\
\cline { 2 - 6 } & Aula & Recreo & Corredores & Aula & Recreo \\
\cline { 2 - 6 } & Gritos & Groserías & & & Empujones \\
\hline \multirow{2}{*}{$\mathbf{N 3}$} & & Groserías & & & \\
\cline { 2 - 6 } & & Insulto & & & Empujones \\
\hline \multirow{2}{*}{ N1 } & Insultos & Groserías & Insultos & & Empujones \\
\cline { 2 - 6 } & Groserías & & Groserías & \\
\hline \multirow{2}{*}{$\mathbf{N 4}$} & \multicolumn{5}{|c|}{ No se registraron actos violentos } \\
\hline
\end{tabular}

Fuente: elaboración propia, 2010.

En los colegios que sirvieron de muestra se observaron que existen manifestaciones de violencia verbal y física. En el aula predomina la violencia verbal, en cambio, en el recreo predomina la violencia física.

Llama la atención que en uno de los colegios no se presenciaron actos de violencia verbal y física. Los alumnos de dicho colegio se dirigían del aula al recreo tanto para la cantina como para realizar sus encuentros lúdicos fueron en forma ordenada y silenciosa. Este hecho podría ser objeto de futuras investigaciones.

A fin de obtener una contextualización de esta problemática, se decide realizar una entrevista a profesores guías de los colegios encuestados.

De diez profesores guías, solo accedieron a la entrevista cinco de ellos, los demás argumentaron contar con poco tiempo para concederla. Teniendo en cuenta que la entrevista era semiestructurada, se procedió a entregar los cuestionarios para retirarlos otro día. Sin embargo, nunca hubo 
devolución de los mismos habiendo ido en reiteradas oportunidades. Los profesores guías entrevistados coinciden en que las conductas físicas y verbales que manifiestan los jóvenes de la educación media son insultos, groserías, ofensas, empujones, patadas y golpes, y que se generan con mayor frecuencia entre varones, pero que cuando se generan entre mujeres, son más intensas y duraderas.

Las situaciones complejas generadas por la conducta violenta de los alumnos se resuelven con medidas alternativas disciplinarias como sanciones, entrevistas a los padres, suspensiones, hasta llegar a la expulsión de la institución.

La autoridad más influyente en el comportamiento de los alumnos es el director del colegio, el profesor guía cuida el orden y podría ser influyente si tiene la suficiente autoridad; muchas veces no es así.

Las situaciones violentas en las que hay mucho descontrol causan angustia; son raros esos casos, pero los profesores guía ya están acostumbrados a manejar la violencia.

Mencionan que cuentan con poco apoyo de los padres en el proceso formativo de los alumnos violentos, concurren a la institución después de varias citaciones y no realizan el seguimiento de la conducta del hijo. En las entrevistas realizadas no han visualizado violencia verbal o física de los padres a los hijos.

Las sanciones y medidas adoptadas por las instituciones dan resultados momentáneos ya que las conductas violentas, pasado un tiempo, vuelven a generarse. Se realizan talleres, reuniones de padres, asistencia espiritual como estrategias para cambiar las conductas de los alumnos y proveer a los padres de herramientas útiles para poder llegar a sus hijos, pero los padres de los alumnos violentos no asisten argumentando falta de tiempo por sus trabajos.

La mayoría de los alumnos violentos no realizan tratamientos con profesionales, otros realizan por períodos breves y una minoría asiste en forma sistemática a terapia, ya que se les solicita constancia del tratamiento, la cual no traen a la institución.

\section{CONCLUSIÓN Y DESAFÍOS PENDIENTES}

Respondiendo a los objetivos de la investigación se puede decir de algunas de las actitudes violentas que manifiestan los padres en forma verbal van 
desde gritar, ordenar y mayormente insultar; mientras que las de violencia física involucran empujones, lanzamiento de objetos, golpes, y en menor frecuencia, hasta amenaza con armas de fuego y blanca.

Las actitudes de violencia verbal que los jóvenes realizan con mayor frecuencia son groserías, insultos, entredichos y desprecios; y las de violencia física son empujones, golpes, robos, amenaza con arma blanca y de fuego.

Con respeco a los estilos de paternidad que con mayor frecuencia generan conductas violentas. En el estilo demócrata, se encontró que no hay protección suficiente de padres a hijos; no hay buena comunicación ya que no hay espacio para el disenso y no existe un adecuado acercamiento de los padres a los hijos. En el estilo autoritario, se encontró que los padres gritan y ofenden a sus hijos, utilizando como estrategia de disciplina el castigo físico y otros en los cuales se exceden. Se evidenció nuevamente la falta de espacio al disenso. En el estilo permisivo, no hay suficiente orientación y supervisión de los padres hacia los hijos; los padres son muy complacientes y los límites son casi inexistentes.

En el estilo democrático no se encontró ningún indicador positivo para la configuración de este estilo, lo que significa que dicho estilo no se relaciona con la conducta violenta de los jóvenes de la muestra.

Con respecto a los indicadores de los estilos autoritario y permisivo, todos ellos resultaron positivos para fomentar la conducta violenta de los jóvenes.

Por otra parte, en cuanto a la percepción que los jóvenes y los docentes tienen acerca de la violencia familiar en los hogares de estos jóvenes. Los jóvenes perciben todo tipo de violencia en el entorno familiar, verbal y física y están imbuidos de conducta violenta en su relacionamiento con los demás. Los docentes perciben que los jóvenes violentos provienen de relaciones intrafamiliares conflictivas en las que se manifiestan todo tipo de violencia, verbal y física.

Los hábitos que incrementan la conducta violenta de los jóvenes se traducen en frecuentar entornos inadecuados fuera del contexto escolar y el consumo de drogas.

Finalmente, se procede a contestar el objetivo general del trabajo.

Quedan, a partir del trabajo investigativo, preguntas que podrían generar nuevos estudios: 
¿A qué se debe el temor tan manifiesto de los componentes de la comunidad educativa a investigar sobre temas tan importantes como la conducta violenta de los jóvenes?

¿Cuál es la relación entre los docentes calificados en el manejo de la violencia y la disminución de la misma en los jóvenes?

¿Se capacita a los padres desde el contexto educativo en el manejo de las conductas violentas de ellos o de sus hijos con técnicas específicas de modificación conductual?

Se espera que en el futuro se realicen trabajos acerca de un tema de vital importancia como es la violencia y que involucre a todos los actores de la comunidad a favor de una educación de calidad en el marco de una sociedad responsable con el futuro del país.

\section{BIBLIOGRAFÍA}

ASTUDILLO, C. y otros; Autoestima Adolescente y estilos de crianza parentales. Recuperado el 3 de julio de 2005 Disponible en:

http://www.geocities.com/heartland/farm8810/investig/acrian2.html.

BLUMER, Herbert. Interaccionismo simbólico Recuperado el 31 de mayo de 2005. http://www.wikipedia.org/wiki/

Campaña Conversatorio Nacional sobre Derechos Humanos (2011). Módulo 3. MEC. www.mec.gov.py

CLARCK, C. y GROSS, K. (2004). Adolescent health-risk behaviors: the effect of perceived Parenting styles and race. Recuperado el 21 de junio de 2005, de http://www.org/Urc/clark.html.

Congreso Nacional (2001). Ley $\mathrm{N}^{\mathrm{o}}$ 1680.Código de la Niñez y la Adolescencia.

CLOSSON, D. (2001). Education: What works? Recuperado el 25 de febrero de 2005, de http://www.probe.org/docs/whatwks.html.

Diccionario de las Ciencias de la Educación (1996).Quinta reimpresión. Madrid, España.

DRESCHER, John. (1996). Siete necesidades básicas del niño. El Paso, TX: Mundo Hispano. Recuperado el 21 de junio de 2005, de www. foroekklesia.com. 
EHRENSA, M.; COHEN, P.; BROWN, J.; SMAILES, E. y CHEN, H. (2003). Intergenerational Transmission of partner violence: A 20-year prospective study. Journal of Consulting and Clinical Psychology, 71(4), 741-756.

ESTRADA, Antonio. (2003). Paternidad, un compromiso con el futuro. São Paulo: Centro Universitario Adventista.

GAXIOLA ROMERO, J. y otros (2006). Validación del cuestionario de prácticas parentales en una población mexicana. Enseñanza e investigación en Psicología, Vol.11, $\mathrm{n}^{\circ}$ 11, 115-128. Recuperado en octubre de 2010, de: http//redalyc.uaemex,mx

GOOD, Thomas y BROPHY, Jere. (1996). Psicología educativa contemporánea. México: McGraw-Hill.

HASTINGS P.; ZAHN-WAXLER, C.; ROBINSON, J. y USHER, B. (2000). Misbehavingc children Concern for others decrease as they enter school. Recuperado el 31 de mayo de 2005, de http://www.apa. org/releases/concern.html.

HAUSER, P.; SIRIN, R. y STIPEK, D. (2003). When teachers and parents values differ: Rating of academic competence in children from low-in families Journal Educational Psychology, 95(4), 813-820.

HERNÁNDEZ SAMPIERI, Roberto (2003). Metodología de la Investigación. México, $3^{\text {a }}$.Editorial: McGrawHill.

HOWSE, K.,DUNTON,H.y MARSHALL,D.(1991). Guía para la dicha familiar. Mountain View, CA: Asociación Publicadora Interamericana.

PAPALIA, Diane; E. WENDKOS OLDS, Sally y DUSKIN FELDMAN, Ruth, (2001). Psicología del desarrollo, Colombia, $8^{a}$ Editorial: McGrawhill.

TAVARES DOS SANTOS, José Vicente. (2001) A violencia na escola: Conflicto social e açoes civilizatorias. Universidad Federal do Rio Grande do Sul.

TAYLOR, C.; CLAYTON, J. y STEPHANIE, J. (2004). Academic socialization: Understanding Parent influences on children's schoolrelated development in the early years. Review of General Psychology, 8(3), 163-178.

TILLER, A.; GARRISON, B.; BENCHEA, E.; CRAMER, K. y TILLER, $V$. (2002). The Influence of parenting styles on children's cognitive 
development. Consultado el 30 de junio de 2005, de http://www.kon.org/ urc/tiller

VALENZUELA, Alfonso. (2002). Cómo fortalecer la familia. Berrien Springs, MI: Promise: Productions.

VALLEJO CASARÍN, A. y LÓPEZ URIARTE, F. X. (2004). Estilos parentales y bienestar psicológico durante la niñez. Revista de Educación y Desarrollo, 2, 83,86.

VERA NORIEGA, J. (2000). Validación de una escala de autoritarismo en madres de una zona rural de México. Vol.14, no 1 y 2, 17-24. 